\title{
Editorial Introduction to the Special Issue: Real-World Applications of THz Systems
}

\section{Masayoshi Tonouchi ${ }^{1}$}

Received: 21 February 2020 / Accepted: 25 February 2020 / Published online: 13 March 2020

(C) Springer Science+Business Media, LLC, part of Springer Nature 2020

Emerging terahertz (THz) technology is now coming into the real world. THz waves locating at frequencies between $100 \mathrm{GHz}$ and $30 \mathrm{THz}$ have been attracting many scientists and engineers for the last two decades in the fields of basic-and-extreme science, nondestructive evaluation, wireless communication, bio/medical/pharmaceutical science and applications, homeland security, and more. In recent years especially, tremendous efforts have been paid for the sake of killer applications. To accelerate such approach, this special issue collects the latest progress on some of the practical applications. It consists of nine invited articles, the content of which is briefly outlined below.

1. "Terahertz-Based Porosity Measurement Of Pharmaceutical Tablets: A Tutorial" by P. Bawuah, D. Markl, D. Farrell, M. Evans, A. Portieri, A. Anderson, D. Goodwin, R. Lucas, and J. A. Zeitler (University of Cambridge, University of Strathclyde, TeraView Ltd., GSK, David Jack Centre, and Huxley Bertram Engineering Ltd.) provides a tutorial for the terahertz-based measurement method that can be used to quantify the porosity of a pharmaceutical tablet within seconds in a nondestructive and noninvasive manner.

2. "Ultrathin Metasurface Wavelength Selective Mirror for Millimeter/Terahertz Wave Fabry-Perot Cavities" by K. Fan, J. Koulakis, K. Holczer, S. Putterman, and W. J. Padilla (Duke University and University of California at Los Angeles) presents an ultrathin metasurface working as both a highly reflective wavelength selective mirror and a hightransmittance output coupler for a semiconfocal Fabry-Perot cavity.

3. "Terahertz Quality Inspection for Automotive and Aviation Industries" by F. Ellrich, M. Bauer, N. Schreiner, A. Keil, T. Pfeiffer, J. Klier, S. Weber, J. Jonuscheit, F. Friederich, and D. Molter (Technische Hochschule Bingen, Fraunhofer Institute for Industrial Mathematics ITWM, and University of Kaiserslautern) gives an overview of state-of-the-art terahertz technology for industrial quality inspection such as three-dimensional imaging of glass fiber-reinforced composites and foam structures, and the characterization of multilayer systems down to some microns and the possibility of measuring the thickness of wet paints.

\section{Masayoshi Tonouchi}

tonouchi@ile.osaka-u.ac.jp

1 Institute of Laser Engineering, Osaka University, 2-6 Yamadaoka, Suita, Osaka 565-0871, Japan 
4. "Optical parameters of gas hydrates for terahertz applications" by K. Takeya, K. Matsumura, R. Takahashi, T. Fukui, S. R, Tripathi, and K. Kawase (Nagoya University and Shizuoka University) reports on the comparison of the frequency dependence and temperature dependence of the optical properties of several hydrogen-bonded solid crystals such as ice, propane hydrate, and methane hydrate in the terahertz wave region.

5. "Electric potential distribution on lithium ion battery cathodes measured using terahertz chemical microscopy" by T. Kiwa, Y. Akiwa, H. Fujita, T. Teranishi, K. Sakai, H. Nose, M. Kobayashi, and K. Tsukada (Okayama University and Kyowa Fine Tech Co., Ltd.) develops a terahertz chemical microscope to visualize electric potential distribution on lithium ion battery cathodes during battery operation.

6. "Evaluation of Rubber Products by Terahertz Time-Domain Spectroscopy - Carbon Black Dispersion and Vulcanization State" by Y. Hirakawa, Y. Yasumoto, and T. Gondo (National Institute of Technology) studies the quality of rubber products by terahertz time-domain spectroscopy.

7. "Experimental Comparison of UTC- and PIN-Photodiodes for Continuous-Wave Terahertz Generation" by S. Nellen, T. Ishibashi, A. Deninger, R.B. Kohlhaas, L. Liebermeister, M. Schell, and B. Globisch (Fraunhofer Institute for Telecommunications, NTT Electronics Techno Corporation, TOPTICA Photonics AG) shows experimental comparison of the two most established optoelectronic emitters for continuous-wave (cw) terahertz generation with a uni-traveling-carrier photodiode (UTC-PD) and a pinphotodiode (PIN-PD).

8. "In-situ determination of the state of conservation of paint coatings on the kiosk of Guadalajara using terahertz time-domain spectroscopy" by F.E.M. Lambert, E.S. ReyesReyes, G.G. Hernandez-Cardoso, A.M. Gomez-Sepulveda, and E. Castro-Camus (Centro de Investigaciones en Optica AC, Universidad de Guanajuato, and Escuela de Conservación y Restauración de Occidente) reports on-site measurements of historically accumulated paint layers on the kiosk of Guadalajara using a terahertz time-domain spectrometer.

9. "Influence of system performance on layer-thickness determination using terahertz timedomain spectroscopy" by S. Weber, L. Liebelt, J. Klier, T. Pfeiffer, D. Molter, F. Ellrich, J. Jonuscheit, and G. Von Freymann (Fraunhofer Institute for Industrial Mathematics ITWM, Technische Universität Kaiserslautern, and University of Applied Sciences) demonstrates the capability of terahertz time-domain spectroscopy to address thickness determination of dielectric single-layer and multilayer coatings on different substrates.

The guest editor would like to thank all contributors and reviewers for their valuable contributions. We hope that the readers will find this special issue useful for their research. 